\title{
Investigation of dielectric properties of multilayer structures consisting of homogeneous plastics and liquid solutions at $75-110 \mathrm{GHz}$
}

\author{
M. Klenner ${ }^{1}$, T. Abels ${ }^{1}$, C. Zech ${ }^{1}$, A. Hülsmann ${ }^{1}$, M. Schlechtweg ${ }^{1}$, and O. Ambacher ${ }^{1,2}$ \\ ${ }^{1}$ Fraunhofer Institute for Applied Solid State Physics (IAF), Freiburg im Breisgau, Germany \\ ${ }^{2}$ Department of Microsystems Engineering (IMTEK), University of Freiburg, Freiburg im Breisgau, Germany \\ Correspondence to: M. Klenner (mathias.klenner@iaf.fraunhofer.de)
}

Received: 29 September 2014 - Revised: 23 February 2015 - Accepted: 3 March 2015 - Published: 19 March 2015

\begin{abstract}
In this paper, we demonstrate an active 3-D millimeter wave ( $\mathrm{mmW}$ ) imaging system used for characterization of the dielectric function of different plastic materials and liquid solutions. The method is based on reflection spectroscopy at frequencies between 75 and $110 \mathrm{GHz}$, denoted as W-band, and can be used to investigate homogeneous dielectric materials such as plastics or layered structures and liquid solutions. Precise measurement of their dielectric properties not only allows for characterization and classification of different fluids, but also for reliable detection and localization of small defects such as voids or delamination within multilayer structures built from plastic materials. The radio frequency (RF) signal generation is based on circuits that have been designed and fabricated at the Fraunhofer Institute for Applied Solid State Physics (IAF) using a $100 \mathrm{~nm}$ InGaAs mHEMT process (Tessmann et al., 2006; Weber et al., 2011).
\end{abstract}

\section{Introduction}

The development of weight- or stability-optimized materials such as plastics or laminates has become the basis of most modern technologies. Manufacturers must meet highest demands in terms of quality, reliability and cost efficiency. Consequently, there is an increasing interest in high-end product surveillance systems that allow for non-destructive material testing at the production stage as well as in operation. For most non-destructive testing methods, either ultrasonics (Blitz and Simpson, 1996) or electromagnetic radiation (Blitz, 1997) are used. The main disadvantage of ultrasonic methods is that a couplant between the transducer and the device under test (DUT) is required (Bourne, 2001), so that they are not suitable for the investigation of large areas. In contrast, remote operation can be realized by using electromagnetic waves. Accordingly, the size of the DUT is not limited, as it can either be moved through a fixed beam or scanned by mechanical or digital beam-forming (Wirth, 2001). Besides visible light, $\mathrm{X}$-rays and terahertz frequencies, the millimeter wave spectrum that spans from 30 to $300 \mathrm{GHz}$ has become well established for non-destructive material analysis.
At the Fraunhofer Institute for Applied Solid State Physics, an active 3-D imaging tomograph operating at frequencies between 75 and $110 \mathrm{GHz}$ (W-band) has been successfully realized. The system can not only be used for detection of concealed objects (Zech et al., 2011), but also for visualization and localization of small defects within multilayer structures consisting of known dielectric materials (Klenner et al., 2013).

In this paper, we present a new experimental setup based on the imaging scanner and investigate the chances and challenges of non-destructive material characterization at $\mathrm{W}$ band frequencies. The method is based on reflection spectroscopy and allows for measurements of the refractive indices of layered plastic samples and determination of the complex dielectric function of different liquid solutions using a Debye model.

\section{Experimental setup}

Figure 1 shows the $\mathrm{mmW}$ imaging scanner operating at frequencies between 75 and $110 \mathrm{GHz}$, denoted as W-band. 


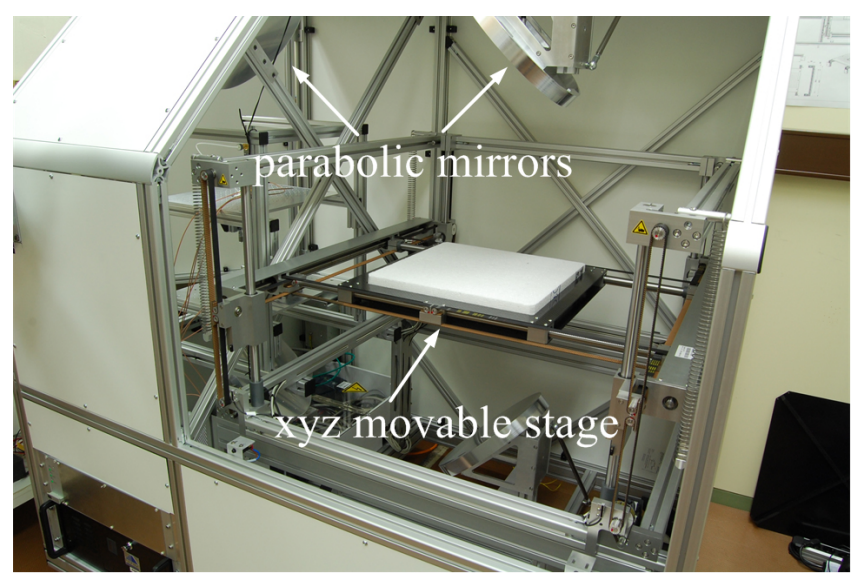

Figure 1. Photograph of the mmW focusing system.

Since the wavelength of W-band frequencies is small enough to approximately describe the signal by quasi-optical ray tracing models, parabolic mirrors have been designed to focus the signal on a movable sample stage. In order to perform reflection spectroscopy on different plastic samples, the system must be able to transmit and receive a signal at fixed frequencies between 75 and $110 \mathrm{GHz}$. This is realized by multiplying the output signals of two HP 8241A synthesizers using in-house $\times 12$ frequency multipliers as shown in the block diagram in Fig. 2. While one of the synthesizers is used to generate the transmit signal, the second one feeds the LO input of an in-house I/Q heterodyne receiver module. Thus, its output frequency is shifted by $\Delta f=50 \mathrm{MHz}$. A Tx \& Rx horn antenna is used for transmission and reception of the mmW beam that is focused at the DUT on the sample stage using parabolic mirrors. A directional Wband coupler $(-10 \mathrm{dBm}$ isolation) is used to feed the RF input of the heterodyne receiver module such that RF and LO signals can be mixed to create in-phase (IF-I) and quadrature (IF-Q) output signals at a fixed intermediate frequency (IF) of $50 \mathrm{MHz}$. Both signals are then amplified and detected using two AD 8310 logarithmic power detectors with a $95 \mathrm{~dB}$ dynamic (DC to $440 \mathrm{MHz}$ ). It turned out that additional low-pass filters are needed to reduce the influence of unwanted system-related interference effects. The setup provides a total bandwidth of $B_{\mathrm{c}}=35 \mathrm{GHz}$ and an output power of $P_{\mathrm{Tx}, \max } \approx 0 \mathrm{dBm}$.

A sketch of two exemplary DUT used for the characterization of different materials is shown in Fig. 3. While homogeneous plastics can be investigated by measuring the reflected intensity of arbitrary multilayer stacks built from several plastic discs, water-based liquid solutions need to be filled in a custom-designed cuvette acting as a Fabry-Perot interferometer.

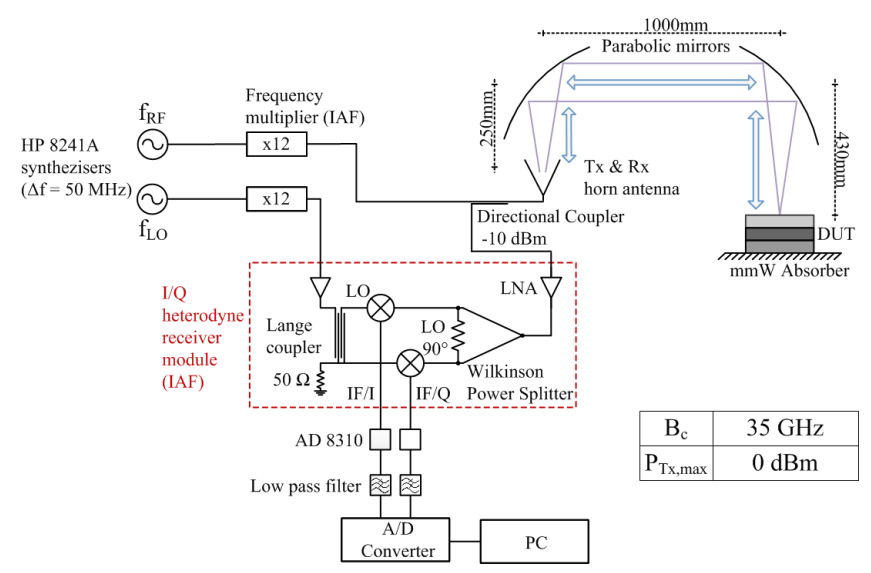

Figure 2. Block diagram of the millimeter wave imaging system used for reflectometric measurements.
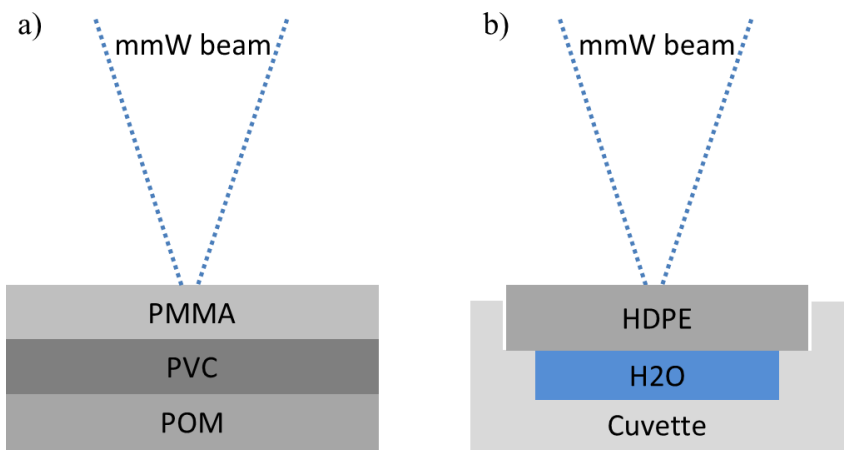

Figure 3. (a) Exemplary three-layer DUT consisting of discs made from POM, PVC and PMMA. (b) Custom-designed cuvette for investigation of liquid solutions such as $\mathrm{H}_{2} \mathrm{O}$. The HDPE layer acts as a Fabry-Perot interferometer.

\section{Data processing}

Since the absorption of millimeter waves in most homogeneous plastics is comparably small (Lamb, 1996), the complex part of the refractive index

$n=n^{\prime}-j n^{\prime \prime}$

can be neglected for investigation of the dispersion of multilayer structures built from homogeneous plastics. For this purpose, theoretical models based on calculation of $\mathbf{S}$ matrices (Kühlke, 2011) are used to fit the measured data in order to obtain the refractive indices of a multilayer sample. Since the experimental setup provides (approximately) perpendicular beam incidence on the sample stage, Fresnel's reflection and transmission coefficients corresponding to a layer $i$ 


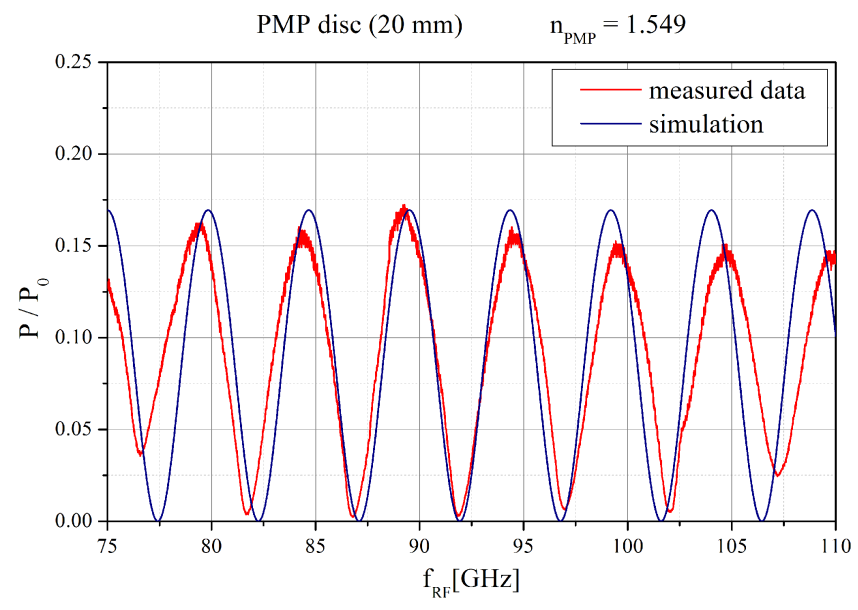

Figure 4. Simulated and measured power spectra corresponding to a $20 \mathrm{~mm}$ thick single-layer PMP sample.

within a multilayer structure are given by

$r_{i-1, i}=\frac{n_{i-1}-n_{i}}{n_{i-1}+n_{i}}$

$t_{i-1, i}=\frac{2 n_{i-1}}{n_{i-1}+n_{i}}$,

where $n_{i}$ is the refractive index of the material in layer $i$. Furthermore, the phase shift of an electromagnetic wave passing through this layer can be calculated as

$\delta_{i}=\frac{2 \pi}{c_{0}} d_{i} n_{i} f$,

where $f$ is the frequency of the transmitted signal, $d_{i}$ is the layer thickness and $c_{0}$ is the speed of light in vacuum. Based on these equations, an $\mathbf{S}$ matrix of the form

$\mathbf{S}=\mathbf{S}\left(n_{0}, n_{1}, \ldots, n_{M}, d_{1}, \ldots, d_{M}, f\right)$

and the corresponding reflectivity

$\rho=\rho\left(n_{0}, n_{1}, \ldots, n_{M}, d_{1}, \ldots, d_{M}, f\right)$

can be derived for arbitrary multilayer structures consisting of $M$ layers. In order to determine the refractive indices of the layers within a sample, the refractive index of the medium surrounding the sample $n_{0}$ as well as the layer thickness $d_{1}, \ldots, d_{M}$ are fixed in the model, while the refractive indices of the layers within the DUT $n_{1}, \ldots, n_{M}$ can be optimized by a Levenberg-Marquardt algorithm (Levenberg, 1944). This model intrinsically assumes that there is no dispersion, as the Levenberg-Marquardt algorithm tries to find a single, optimal refractive index per layer for the full W-band. Therefore, dispersion will qualitatively become visible as a discrepancy between model and experimental data, as shown in Fig. 4.

In order to investigate material dispersion in a more quantitative way, it is necessary to select narrow frequency ranges rather than the full W-band to fit the model more accurately. The following method can be used.
1. Fit the experimental data for the full $\mathrm{W}$-band to obtain a refractive index $n_{i}^{\text {Full }}$ for every layer $i$.

2. Define upper and lower limits for possible values of the refractive indices based on $n_{i}^{\text {Full }}$. This will increase the convergence rates of the following fits.

3. Identify the first maximum or minimum and set a corresponding counter variable to $k=0$.

4. Select a narrow frequency range around the $k$ th extremum in the measured spectrum.

5. In consideration of the upper and lower limits defined in step 2, fit the measured data within the narrow range to obtain refractive indices $n_{i}^{k}$.

6. Slightly vary the values of $n_{i}^{k}$ (manually) until the model does significantly deviate from the measured data. This allows for estimation of the errors in $n_{i}^{k}$.

7. Identify the center frequency $f_{m}^{k}$ of the maximum or minimum and estimate its uncertainty $s_{f}^{k}$.

8. Save data point $\left(f_{m}^{k}, n_{i}^{k}\right)$ and corresponding error estimations.

9. Select the next extremum $(k=k+1)$.

10. Repeat steps 4 to 9 for every maximum and minimum in the spectrum.

The first two steps are mandatory for obtaining reasonable results from the Levenberg-Marquardt algorithm when only a narrow range of measurement data is used. In particular, there is no unique solution for multilayer DUT, and the algorithm does not converge properly without limiting the fitting parameters. In addition, it is useful to define a measure of inaccuracy for the fitted model such as the following sum of squares:

$\sigma=\sqrt{\frac{1}{N} \sum_{j=1}^{N}\left(P_{j}-T\left(f_{j}\right)\right)^{2}}$.

$P_{j}$ is the $j$ th of $N$ measured values and corresponds to the frequency $f_{j}$. Accordingly, $T\left(f_{j}\right)$ is the value of the theoretical model at $f_{j}$. At the first step of the method described above, $\sigma$ can be used for a first qualitative characterization of the strength of dispersion of a material over full W-band. It is, however, neither a quantitative measure of the relaxation strength nor a confidence limit on the refractive indices. Since there is only a slight dispersion in most materials that have been investigated, one could also use a model containing an additional distribution parameter for the refractive index instead of the method described above. In the case of a single-layer sample, the convergence rate of a model considering dispersion is still sufficient, while it is significantly 
decreasing when analyzing two- or three-layer DUT. Therefore, the algorithm described above is, in general, superior regarding convergence rates.

In principle, the analysis of liquid samples can be performed in a similar way. However, the assumption of negligible contribution of the complex part of the dielectric function no longer holds for water solutions, as they are significantly absorbing in the mmW regime (Peacock, 2009). Consequently, the algorithm presented above leads to significant uncertainties. In order to reliably fit the measured data using a model where dispersion is parameterized, the lid of the cuvette (cf. Fig. 3) must be made of a material with very weak dispersion such that the sample only consists of one layer where dispersion must be considered. This is realized by using PMMA and HDPE lids and a Debye-type model of the form

$\epsilon(\omega)=\epsilon^{\prime}(\omega)-\mathrm{j} \epsilon^{\prime \prime}(\omega)=\epsilon_{\infty}+\frac{\epsilon_{0}-\epsilon_{\infty}}{1+\mathrm{j} \omega \tau}$

to parameterize the complex dielectric function of the liquid layer. Hereby, $\epsilon_{0}$ and $\epsilon_{\infty}$ describe, respectively, the lowand high-frequency limits of the dielectric function. The parameter $\tau$ is the relaxation time of the dipole polarization process within the liquid solution caused by an incident electromagnetic field. Since a custom-designed cuvette is used for measurements of solutions (cf. Fig. 3), the layer structure of different DUTs is always identical and the sample can be regarded as a Fabry-Perot interferometer. Hence, instead of using an $\mathbf{S}$ matrix formalism, the reflected intensity from the cuvette can be described by the simpler relation

$\frac{I_{r}(\omega)}{I_{0}(\omega)}=\left|r_{01}+t_{01} r_{12} t_{10} e^{-2 i \delta} \sum_{j=0}^{\infty}\left(r_{10} r_{12} e^{-2 i \delta}\right)^{j}\right|^{2}$,

where

$r_{12}(\omega)=\frac{n_{1}-\sqrt{\epsilon_{\infty}+\frac{\epsilon_{0}-\epsilon_{\infty}}{1+i \omega \tau}}}{n_{1}+\sqrt{\epsilon_{\infty}+\frac{\epsilon_{0}-\epsilon_{\infty}}{1+i \omega \tau}}}$

is the reflection coefficient corresponding to the interface with the liquid solution, which is described by the Debye model, and $\delta$ is the phase shift of the electromagnetic field within the material on top of the liquid (cf. Eq. 3). By optimization of the Debye parameters $\epsilon_{0}, \epsilon_{\infty}$ and $\tau$, the model can be fitted to the measured data to obtain the evolution of the complex dielectric function of liquid solutions at W-band frequencies.

\section{Dispersion of layered plastics}

Table 1 shows the results of fitting theoretical models describing the reflectivity of single discs of POM, PVC,
Table 1. Average refractive indices of different plastics at W-band frequencies.

\begin{tabular}{lcc}
\hline Material & $n^{\text {Full }}$ & $\sigma^{\text {Full }}$ \\
\hline Polyoxymethylene (POM) & 1.752 & 0.055 \\
Polyvinyl chloride (PVC) & 1.703 & 0.057 \\
Polymethyl methacrylate (PMMA) & 1.590 & 0.027 \\
Polymethylpentene (PMP) & 1.549 & 0.053 \\
High-density polyethylene (HDPE) & 1.512 & 0.021 \\
\hline
\end{tabular}

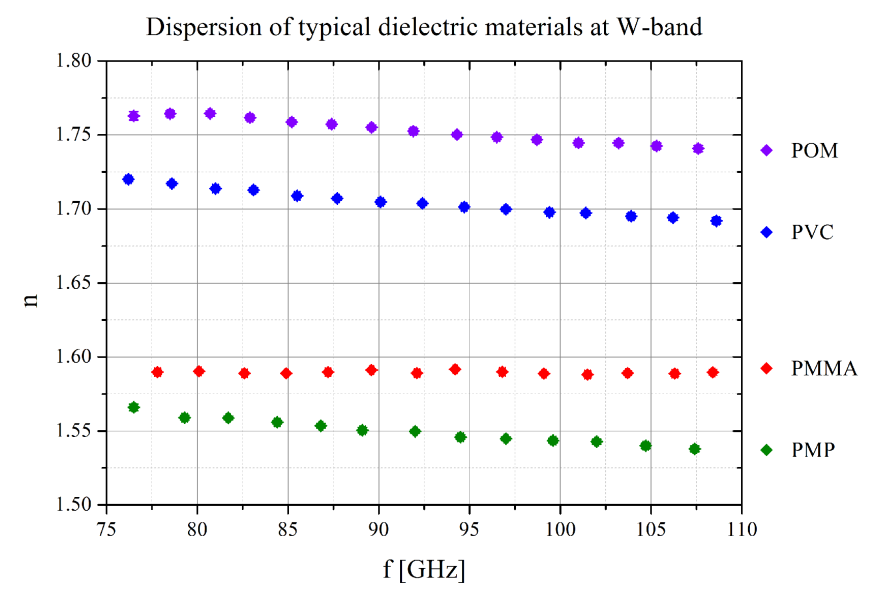

Figure 5. Dispersion of different plastics measured from single discs.

PMMA, PMP and HDPE (each $d=20 \mathrm{~mm}$ thick) to the measured data. The refractive indices $n^{\text {Full }}$ have been found by optimization using a Levenberg-Marquardt algorithm. Additionally, the confidence limits $\sigma^{\text {Full }}$ of the respective optimized model are shown for each refractive index. Note that this is not an explicit error of $n^{\text {Full }}$ but a measure of how well the model, which assumes constant refractive indices, describes the corresponding experimental data that are in general influenced by dispersion. Assuming that the measurements have been performed under similar conditions, a relative difference in $\sigma^{\text {Full }}$ between two materials is therefore essentially caused by their different strengths of dispersion.

By using the method described in Sect. 3, the refractive indices of narrow regions around the maxima and minima of the measured interference spectra (see Fig. 4) have been determined for each of the four plastic discs. The optimization algorithm did converge within all the selected regions such that a refractive index could be determined at every extremum. The resulting frequency-dependent refractive index is plotted for all the discs in Fig. 5. While the refractive indices of POM, PVC and PMP slightly decrease over Wband, PMMA shows nearly no dispersion. Thus, the dispersion plots are in agreement with the data shown in Table 1.

Multilayer samples have been investigated by building arbitrary stacks consisting of two, three or four discs that are either 10 or $20 \mathrm{~mm}$ thick. An exemplary dispersion plot cor- 
(a)
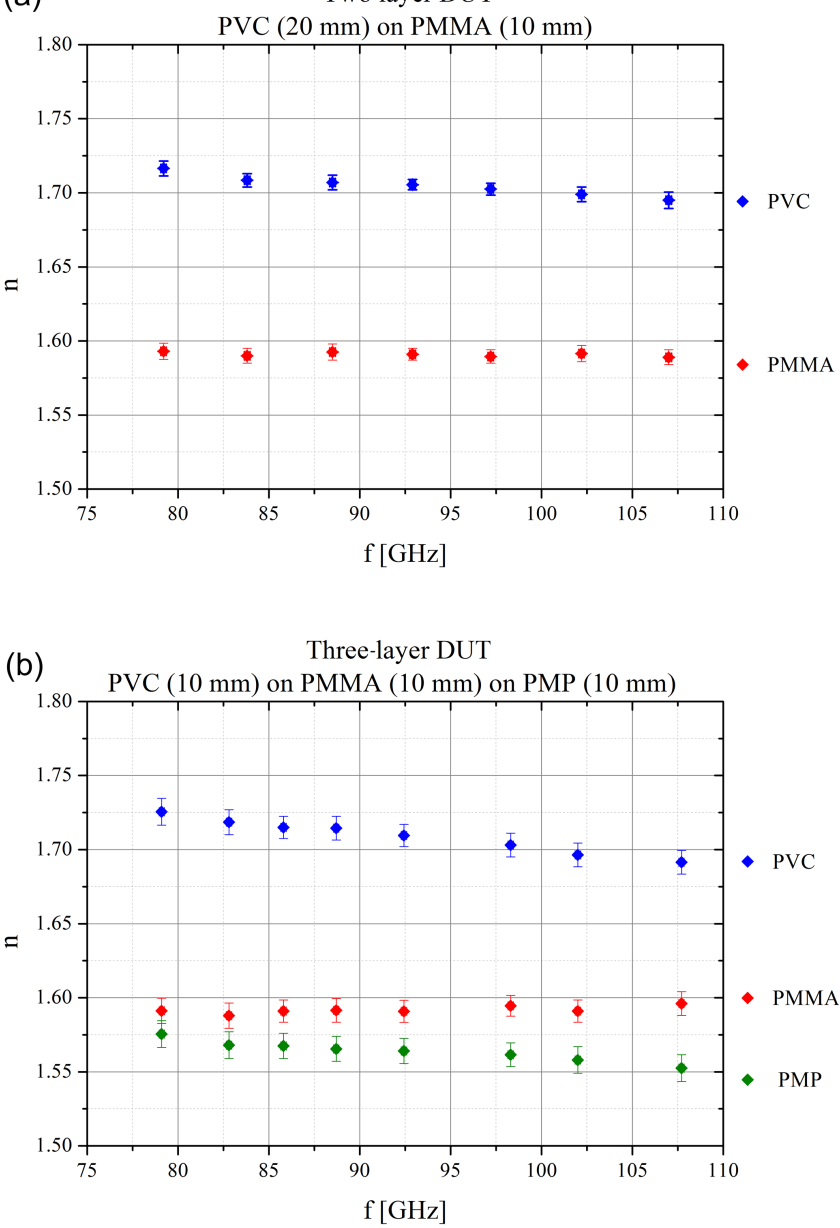

Figure 6. (a) Dispersion of different plastics measured from a double-layer structure. (b) The refractive index of PMP measured from a three-layer sample is overestimated compared to the singledisc measurements.

Table 2. Conductance and $\mathrm{pH}$ values of the different solutions.

\begin{tabular}{lrr}
\hline DUT & Conductance $\left(\mathrm{mS} \mathrm{cm}^{-1}\right)$ & $\mathrm{pH}$ value \\
\hline DI water & 0.1 & 7.9 \\
HCL solution & 740.0 & 0.4 \\
$\mathrm{NaCl}$ solution & 171.2 & 8.2 \\
$\mathrm{NaOH}$ solution & 401.0 & 12.6 \\
\hline
\end{tabular}

responding to a two-layer stack is shown in Fig. 6a. Since the power spectra become more irregular with the number of layers, frequency resolution of the dispersion plots is decreased compared to the single-disc measurements. However, both measurements are in agreement, and this is also true for any other combination of two discs.

Measurements of three- and four-layer samples have shown that, in several configurations, the refractive indices of deeper layers are overestimated with respect to the single-
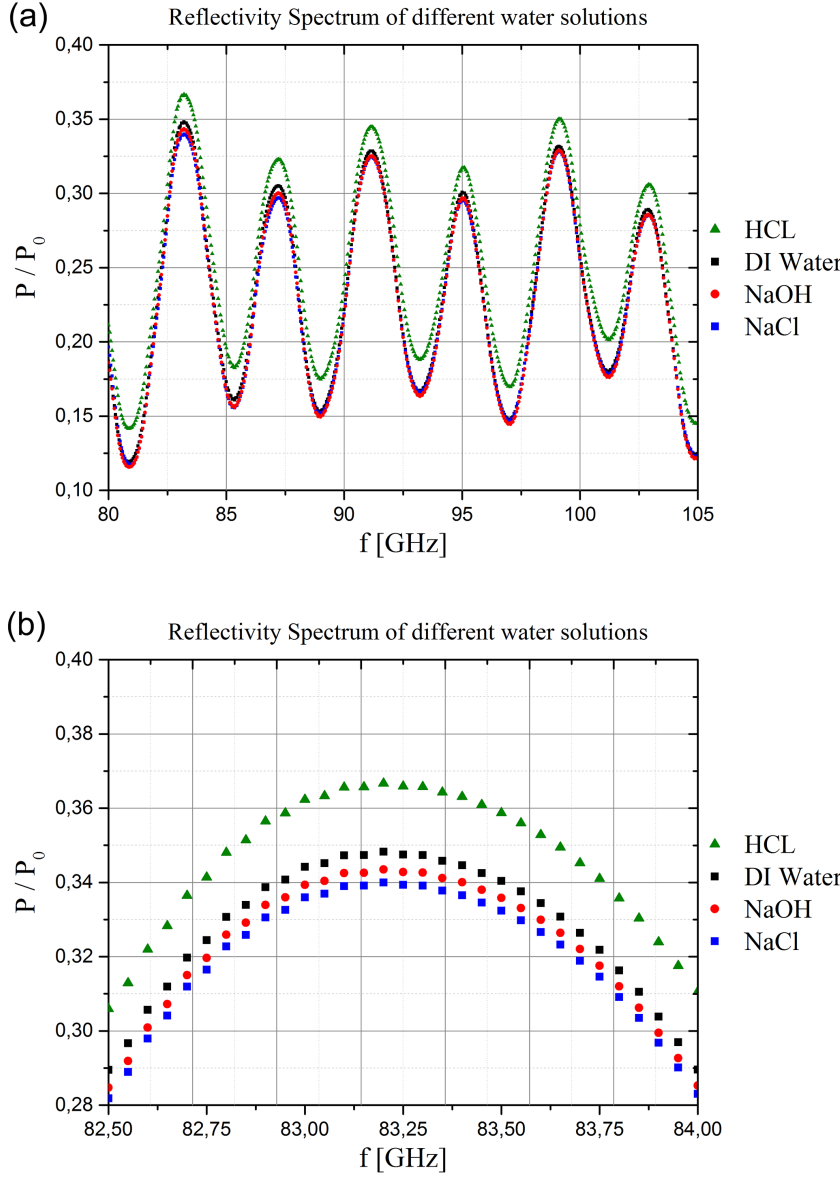

Figure 7. (a) Reflectivity spectrum of a HDPE cuvette filled with different solutions. (b) Error bars on the data points are much smaller than the markers used for visualization. Therefore, the different solutions are clearly distinguishable.

layer measurements. This behavior occurs if the refractive indices of upper layers are larger than in lower layers. Figure $6 \mathrm{~b}$ shows the dispersion curves obtained by measuring the reflectivity of a three-layer sample where the lowest layer is a PMP disc and the corresponding refractive index is overestimated compared to the measurements shown in Fig. 5. The overestimation is most probably caused by the assumption of perpendicular incidence on the sample, which is not entirely true. In particular, beams get refracted away from the perpendicular in deeper layers if there are primarily transitions from a medium of higher refractive index to a medium of lower refractive index. Furthermore, parts of the signal get refracted out of the beam path of the system, causing a lower convergence rate of the Levenberg-Marquardt algorithm and therefore larger error bars compared to the single-disc measurements. 


\section{Complex dielectric function of water solutions}

In order to investigate whether water solutions of different physical or chemical properties can be uniquely distinguished using reflection spectroscopy at W-band frequencies, a set of four sample solutions based on DI water has been prepared. By measuring their conductances and $\mathrm{pH}$ values, the solutions have been characterized as shown in Table 2. For the measurements, each solution is filled in a custom-designed cuvette with a $25 \mathrm{~mm}$ thick top layer made of HDPE. Accordingly, dispersion of the top layer can be neglected at W-band frequencies. Due to the finite isolation of the directional coupler used in the setup, a high-frequency interference pattern overlays the raw data of the spectra. Thus, a Savitzky-Golay algorithm (Savitzky and Golay, 1964) is used to smoothe the data sets. Furthermore, the reflectance of each DUT is measured ten times in succession to determine the reproducibility of the spectra by averaging and calculating the standard deviation. Figure 7 shows the smoothed and averaged reflection spectra of cuvettes filled with pure DI water as well as $\mathrm{HCL}, \mathrm{NaCl}$ and $\mathrm{NaOH}$ solutions. The standard deviation of the curves is on the order of $10^{-5}$. Error bars are therefore much smaller than the markers used for visualizing the data. Accordingly, reflection spectroscopy at W-band frequencies can be reliably used to detect or uniquely differentiate solutions of different conductances or $\mathrm{pH}$ values.

The complex dielectric function of these solutions can be calculated using the Debye model represented by Eq. (7). Thus, the parameters $\epsilon_{0}, \epsilon_{\infty}$ and $\tau$ must be determined from the measured data sets. Therefore, the reflection coefficient corresponding to the liquid interface is parameterized by the Debye model (cf. Eq. 9) so that a Levenberg-Marquard algorithm can be used to fit Eq. (8) to the measured data by optimization of the Debye parameters.

However, simulations have revealed that all parameters influence the spectra in a similar way and that $\tau$ is by far the most sensitive parameter (Abels, 2014). These theoretical results are in accordance with other investigations showing that the presence of foreign molecules in water mainly result in the reduction of the potential barrier of the dipole reorientation process or an enhanced formation of hydrogen bonds (Kaatze et al., 2002). Both effects directly influence the dipole relaxation time and therefore the parameter $\tau$. Accordingly, the convergence rate of the Levenberg-Marquardt algorithm is low without weighting or limits on the Debye parameters. In order to take this effect into consideration, the Debye parameters are first determined for a pure DI water solution. The results are in good accordance with other values found in the literature (Peacock, 2009), so that the weights of the optimization process can be calibrated with respect to the DI water sample. Assuming that the presence of HCL, $\mathrm{NaOH}$ or $\mathrm{NaCl}$ molecules in the water solution mainly influences the relaxation time $\tau$, the dielectric function has been determined for each of the samples as shown in Fig. 8. While the dielectric function corresponding to the basic solutions is (a)

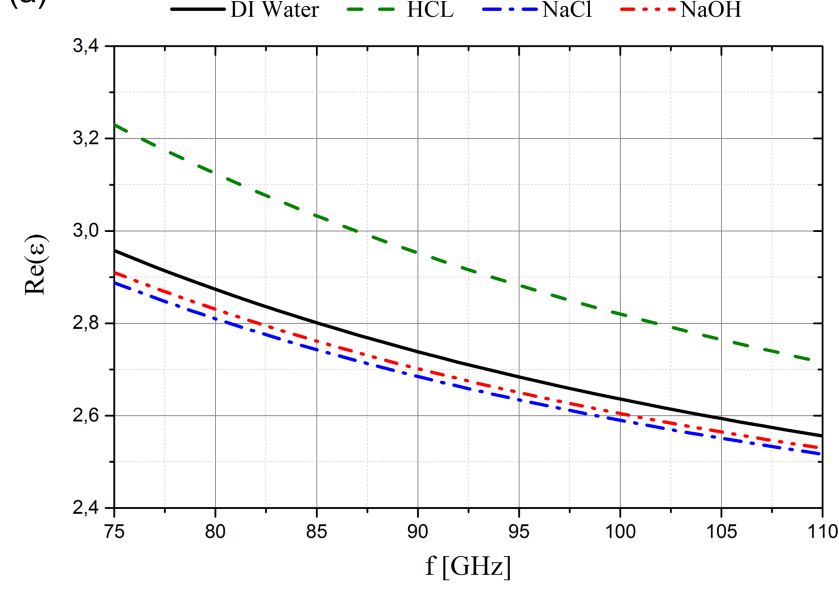

(b)

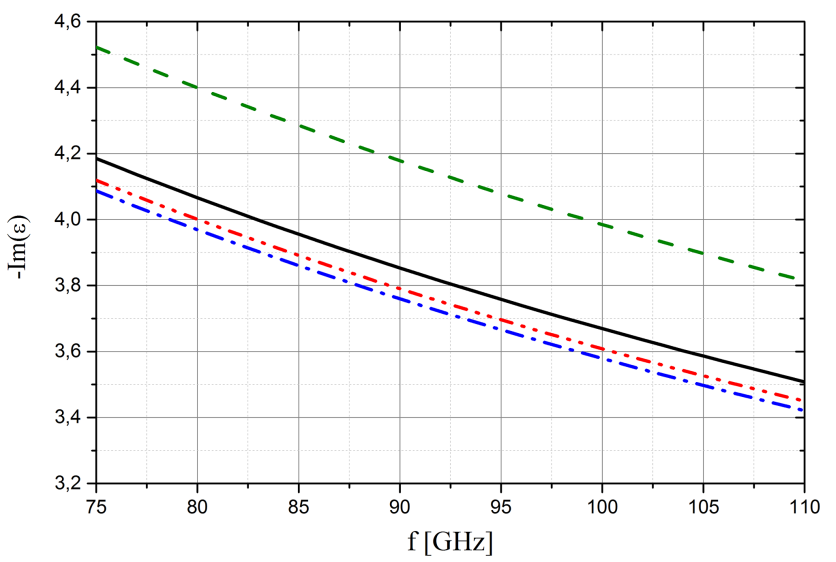

Figure 8. Real and imaginary parts of the dielectric function of different water solutions. Due to measurement inaccuracies, the dielectric function of the $\mathrm{NaCl}$ and $\mathrm{NaOH}$ solutions can not be uniquely distinguished.

lower compared to DI water, the permittivity spectrum of the acidic HCL solution is increased. These results are in contrast to prior investigations at frequencies of up to $40 \mathrm{GHz}$ (Kaatze, 1983), where the real part of the dielectric function of hydrochloric acid solutions is lower compared to pure water.

A dielectric decrement can be attributed to the specific conductivity of the acidic solution. If this decrement is strong enough to significantly superimpose the relative increase of the dielectric function caused by its relaxation time, the above assumption no longer holds and a more detailed model must be considered to fit the measured data. Even though, to the authors' knowledge, there are no comparable measurements in the frequency range between 75 and $110 \mathrm{GHz}$, it appears likely that this is the case. However, due to the necessity of strong smoothing of the spectra, which influences both the amplitude and position of peaks, the use of more complex models is not feasible using the current experimen- 
tal setup, and uncertainties in the parameters of the fitted Debye model are already significant. Hence, $\mathrm{NaOH}$ and $\mathrm{NaCl}$ solutions are not uniquely distinguishable.

\section{Conclusions}

We have demonstrated a reflectometric setup to measure the refractive indices and dispersion of different multilayer samples made from homogeneous plastics as well as the Debye parameters corresponding to a relaxation model describing the complex dielectric function of liquid solutions. The setup is included in a mmW imaging scanner so that the information on the dielectric behavior of different DUT can be directly used for $\mathrm{mmW}$ imaging.

Small defects in multilayer structures have been successfully visualized and localized based on the data obtained by reflectometry measurements (Klenner et al., 2013). In general, the presented method works well with stacks consisting of two or three layers. However, the refractive indices of lower layers in several configurations of three- and four-layer samples are overestimated if the signal gets significantly refracted away from the perpendicular. The performance of the system is strongly dependent on the convergence rate of the Levenberg-Marquardt algorithm and can be increased if some material parameters are already known. Accordingly, the method can for example be used to detect specific materials within unknown multilayer stacks, in particular if the dielectric properties of the target material adequately differ from its surroundings.

Furthermore, the reflectances of acid and basic solutions have been investigated and compared to pure DI water. While the reflectance spectra of all the samples are clearly distinguishable with respect to statistical uncertainties, the strong parasitic interference caused by the directional coupler used in the setup leads to significant inaccuracies regarding the model parameters when the data are fitted. As a consequence, more complex models could not be investigated in more detail. In addition, samples that only differ slightly in their dielectric behavior, such as the two basic solutions that have been investigated, can not be uniquely distinguished.

Acknowledgements. The authors would like to thank the workshop of the Fraunhofer IAF for precise mechanical prototype fabrication of the parabolic mirrors used in the imaging system and the dielectric samples.

Edited by: R. Lucklum

Reviewed by: two anonymous referees

\section{References}

Abels, T.: Reflexionsspektroskopie an verschiedenen Wasserproben im Millimeterwellenbereich, Bachelor Thesis, University of Freiburg im Breisgau, Germany, 44 pp., 2014.

Blitz, J.: Electrical and magnetic methods of non-destructive testing, Chapman \& Hall, London, New York, USA, 1997.

Blitz, J. and Simpson, G.: Ultrasonic methods of non-destructive testing, Chapman \& Hall, London, UK, 1996.

Bourne, S.: Novel hydrophilic polymer couplant for application in ultrasonic non destructive testing, PhD Thesis, Cranfield University, UK, 2001.

Kaatze, U.: Dielectric Effects in Aqueous Solutions of $1: 1,2: 1$, and $3: 1$ valent Electrolytes: Kinetic Depolarization, Saturation, and Solvent Relaxation, Zeitschrift für Physikalische Chemie, 135, 51-57, 1983.

Kaatze, U., Behrends, R., and Pottel, R.: Hydrogen network fluctuations and dielectric spectrometry of liquids, J. Non-Cryst. Solids, 305, 19-28, 2002.

Klenner, M., Zech, C., Hülsmann, A., Tessmann, A., Leuther, A., Schlechtweg, M., Wagner, J., and Ambacher, O.: Multilayer material analysis using an active millimeter wave imaging system, in: 14th International Radar Symposium (IRS), Dresden, Germany, 19-21 June 2013, 1, 207-213, 2013.

Kühlke, D.: Optik - Grundlagen und Anwendungen, EuropaLehrmittel, Edition Harri Deutsch, Frankfurt am Main, Germany, 407 pp., 2011.

Lamb, J. W.: Miscellaneous data on materials for millimetre and submillimetre optics, Int. J. Infrared Milli., 17, 1997-2034, 1996.

Levenberg, K.: A method for the solution of certain non-linear problems in least squares, Q. J. Appl. Math., II, 164-168, 1944.

Peacock, J. R.: Millimetre wave permittivity of water near $25^{\circ} \mathrm{C}$, J. Phys. D-Appl. Phys., 42, 205501, doi:10.1088/00223727/42/20/205501, 2009.

Savitzky, A. and Golay, M. J. E.: Smoothing and Differentiation of Data by Simplified Least Squares Procedures, Anal. Chem., 36, 1627-1639, 1964.

Tessmann, A., Kuri, M., Riessle, M., Massler, H., Zink, M., Reinert, W., Bronner, W., and Leuther, A.: A Compact W-Band DualChannel Receiver Module, in: Microwave Symposium Digest, 2006, IEEE MTT-S International, San Francisco, CA, USA, 1116 June 2006, 85-88, 2006.

Weber, R., Lewark, U., Leuther, P., and Kallfass, I.: A W-Band 12 Multiplier MMIC With Excellent Spurious Suppression, IEEE Microw. Wirel. Co., 21, 212-214, 2011.

Wirth, W.: Radar Techniques Using Array Antennas, 2nd Edition, IET - The Institution of Engineering and Technology, 560 pp., 2001.

Zech, C., Hülsmann, A., Kallfass, I., Tessmann, A., Zink, M., Schlechtweg, M., Leuther, A., and Ambacher, O.: Active millimeter-wave imaging system for material analysis and object detection, in: Millimetre Wave and Terahertz Sensors and Technology IV, Proceedings of SPIE 8188, 81880D, 2011. 